\title{
Effective Student Teams: A Faux Hiring And Peer Evaluation Process
}

\author{
Michelle D. Lane, Western Kentucky University, USA
}

\begin{abstract}
Student team projects provide benefits to the education process and provide experience valued by employers, but they can also be a source of conflict, free-riding and are fraught with 'fairness' issues. The advantages of using teams for faculty are that they provide opportunities for synergies and collaboration, while also simulating group work processes. However, due to free-riding, scheduling problems and differing goals, there are fertile grounds for team conflicts. Therefore, there exists a need for better methods of forming teams and a process to assure shared goals by team members. This research proposes an innovative approach to team creation where students participate in a 'hiring' process that increases team cohesiveness and ultimately team performance. Preliminary empirical validation is also provided that supports this approach.
\end{abstract}

Keywords: Team Formation; Student Teams; Group Selection

\section{INTRODUCTION}

$\mathscr{Q}$ se of teams in business is prevalent and the need for work teams is clear. Businesses benefit from the synergies and communications that result from team interactions. If the teams are effective, the firm experiences increased efficiencies, long-standing productive relationships and a more cohesive organization culture. Research has shown that the majority of job failures can be attributed to people skills (85\%) while only $15 \%$ is due to proficiency (Arien, 2001). This substantiates the importance of team dynamics. The culture within the team that supports positive team interactions will help create a supportive and open environment (Winter, Waner and Neal-Mansfield, 2008). Therefore, the communication processes that are used by student teams during their formation and operation are critical to their successful performance and are the focus of this research.

Post-secondary education has adopted team-based approaches to class work both to develop student skills in teamwork and to tackle larger projects than a single student can handle during a semester. Unfortunately, any time you get a group of people together as a team to accomplish some defined objective, there is an opportunity for conflict. This conflict may result in unsatisfactory team outcomes and perhaps create a long-standing negative view of teamwork. Therefore, as educators, a methodology is needed that can be used to form student teams that will help in reducing the risk of a negative experience and help them build team skills that they can carry into the workplace. This research presents a methodology and empirical analysis for team formation whereby students form teams through a simulated hiring process. This allows for teams to form that share the same goals and objectives and have common interests. It also gives them experience in self-promotion and simulated 'hiring' of team members. A literature review is provided in the next section. The process for team formation is then delineated and preliminary empirical support for this process is then provided.

\section{REVIEW OF THE LITERATURE}

The use of teams by educators is a commonly accepted practice in higher education. Employers expect their employees to be 'team players' as they have found substantial benefits from effective teamwork (Ashraf, 2004; Chen, Donahue and Klimoski, 2004). Teamwork has been shown to improve the knowledge, skills and abilities of the team members (Chen et al., 2004). Other benefits include increased skills in conflict resolution, collaborative problem solving, communication, goal setting, performance measurement and task coordination (Stevens and Campion, 1994). Therefore the justification for team training in the classroom is clear. Students who are adept at 
working in teams will be better prepared for their work environment and should be more effective in accomplishing employer goals.

Teams are used in higher education to benefit the employers but the benefits of group projects are also important as a means of active learning rather than passive lecture based methodologies. Students can also take on larger endeavors by sharing the workload that would be impossible for one student to undertake. From an instructors viewpoint it may also be a course manageability decision in cases where large class sizes preclude the assignment of large projects at the individual level. Assigning a large number of individual students to study local businesses or tackle large complex projects would be impossible to monitor, supervise and grade. Grouping the students into teams makes these projects feasible to manage. The use of teams can provide significant benefits to the students as it simulates real business team working experiences and increases competitiveness (Winter et al., 2008; Young and Henquinet, 2000). Team interactions have been shown to enhance their learning through the sharing of diverse ideas which improves creativity and increases learning by doing (Winter et al., 2008). The synergistic team processes including planning, debating and considering the ideas of their teammates provides them experience with teamwork skills employers need (Fish, 2007; Koppenhaver and Shrader, 2003; O’Connor and Yballe, 2007).

There is a downside to consider when using teams for the purposes of earning a grade. Research has shown that student teams are disliked more than work teams (Connerly and Mael, 2001). There are two major reasons college students dislike the use of teams. First free-riding students tend to earn grades that are better than they would earn on their own, since they are not held accountable individually. On the other hand, students that carry more than their share of the team workload may earn grades lower than they would if they worked alone (Bacon, Stewart and Silver, 1999; Brandyberry and Bakke, 2006; Wilcoxson, 2006). Second, team members may be uncooperative and not willing to adhere to the goals and processes adopted by the rest of the team (Brandyberry and Bakke, 2006). Team tensions caused by inaccurate assessments, scheduling difficulties, goal differences and embarrassing comments are common complaints (Williams and Anderson, 2008). These team failures revolve around the composition of the team, the communication within the team and with the instructor and the weaknesses of the team member evaluation process.

The team formation process is always a source of considerable concern for educators. In courses where team outcomes are a large proportion of students' grades, successful team selection is of major importance. The difficulty in selecting student teams for instructional projects is well documented (Koppenhaver and Shrader, 2003; McClosky, 2004). If the instructor chooses the teams, the students may complain that they did not have any input and were 'stuck' with someone who did not fit with their goals or methods. If the students get to select the team, then they are usually in one of two camps: 1) Teams that forms based on physical proximity in class where students do not know each other or 2) Students choose friends who may not have the same aspirations and work ethic. Self selection into a team has been shown to yield better team cohesiveness that random assignment and teams with a longer time to work together plus a well-defined task perform better (Bacon et al., 1999). The importance of the 'fit' of the student within their team is a contributor to team success. The team members should take time to interact and support each others ideas. The team longevity also increases the likelihood of their success when they have the time to get to know each other (Hansen, 2006; Winter et al., 2008). It has also been found that mixed majors develop more friendships and feel more comfortable expressing themselves to the group lending support for group heterogeneity (Winter et al., 2008). Perhaps students evolve better into an 'expert role' in their area without other in the same major the challenge them.

The information available for team formation is also shown to be very important in team performance (Connerly and Mael, 2001). The information shared should be relevant to the task to be accomplished but not too invasive to cause concern. Students' work ethic, time availability, course goals and attitude toward class projects are important information that helps in the selection process. This research suggests that arranging heterogeneous teams where students have some input into the process and knowledge about their classmates should perform better than randomly assigned teams (Bacon et al., 1999; Connerly and Mael, 2001).

Therefore, this research addresses the team formation process using a "faux hiring" of student team members is a means to reduce or eliminate many of these problems. The steps in the faux hiring process will be summarized and empirical evidence from test and control groups will be presented. 


\section{THE TEAM SELECTION PROCESS}

Figure 1 shows the steps in the faux hiring process that will be discussed subsequently. There are two channels used to effectively apply this methodology. First, there is the flow of the process activities, from posting resumes, stating personal course goals, interviewing, team selection and finally, developing a contract. The communications flows are the other component to the model and reflect the importance of information that is available to the team at all times during the process, including their resume and class goals, the contract, and finally, the peer evaluation.

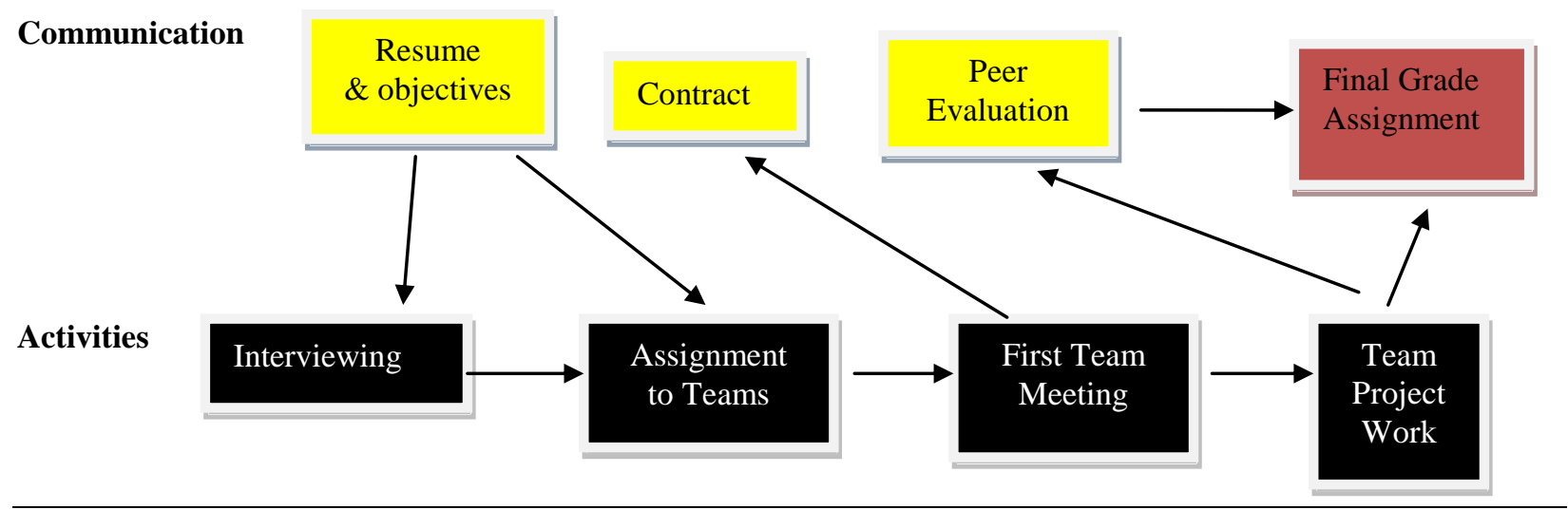

Figure 1: The Faux Hiring Team Selection Process

The process of team selection and formation involves the following six steps:

1. Selection of interviewers

2. Posting interviewee's applications for review by classmates and interviewers

3. Holding a 'Job Fair' where a faux interview process occurs

4. Selection of personal top choices by both interviewers and interviewees

5. Instructor team assignment

6. Forming and signing the team contract

Interviewer selection can be done using many methods. In the business plan entrepreneurship class, each student presents their entrepreneurial business idea to the class and the class votes on the ideas that are most likely to be successful. The 'idea champions' for the ideas with the most votes then become the interviewers recruiting people for their business plan team. In an operations management class, where local business operations are studied, students each propose a local business that they want to study, and the class votes on the businesses they would like to be examining. The top businesses are selected for study and the person who proposed that business will be the 'company representative' hiring people for their business study team. If there is no specific business focus in the course, usually just asking for volunteers to be the interviewers is successful. There are enough students who want to have more control of their own destiny so getting enough interviewers has not been difficult. If there is need to select interviewers this is done this based on knowledge of their class participation abilities.

The second step in the process requires the students, including interviewers, to post their resume and course objectives online. This is not a complete 'resume' but rather a document to self-promote and clarify their team experiences and course goals. This can accomplished through a discussion board or class wiki and contains information about themselves and their major, career goals, expected grade in the class, leadership ability, and good and bad past team experiences. Everyone in the class is to review this information before the job fair. There is quite a bit of variation between students in the amount of effort that they put into this application which is also indicative of their attitude about teamwork. 
On job fair day, the entire 80 minute class period is dedicated to interviews when there are 20 to 30 students. The interviewers sit at various tables spread out around the classroom. The students are allowed in the room, one for each interviewer and they spend three to four minutes talking with the interviewer before I ring a bell and tell them to rotate to another position. Students are allowed to walk around the interview area and talk to any interviewers that they are interested in similar to a traditional job fair. The interviewees do not have to talk to all interviewers but they do need to talk with at least three. Eventually the entire class rotates through the interview stations that they are interested in. They are encouraged to consider a mix of talents for their teammates and this is part of the focus of their discussions.

After the job fair the students will express their interest in each of the interviewer teams providing 3 points to their top choice, 2 points to their second choice and one point to their third choice. Interviewers will also submit rankings for their top teammate choices ranking them from high to low (the number of requests should be at least $150 \%$ of the ending team size). These rankings are submitted within 24 hours of the job fair. Students may also request to not be assigned to a certain interviewer.

The team assignment then takes place, matching up the highest ranking requests. These are posted online before the next class period. The students are assigned to teams based on the point scoring system used by both the interviewers and the students. A matrix of the points assigned by each student with each interviewer is used. Usually everyone gets either their first or second choices. In selecting the team members, the variety of the student skills are also considered in order to obtain a mixture of talents. Since there are a multitude of possible combinations obtaining team variety and honoring requests this can usually be accomplished. The teams meet for the first time in the next class meeting after the job fair. They are given an opportunity to get to know one another and then they discuss and agree upon a team contract. For purposes of this study, there were six control group teams of six to seven students which were randomly assigned and six experimental group teams of the same size using the above technique, to be able to determine the impact of the job fair selection method. The characteristics of effective teams included in the contract were taken (2007). The team contract is drawn up and was based primarily on the contracted presented in O'Connor and Y'Balle's 2007 research.

\section{RESULTS}

In order to evaluate the effectiveness of the faux hiring process, two classes of 40 students each were studied in the fall semester 2009. Independent T-Tests showed no statistical difference between the two classes in terms of age and GPA. In the first class, the teams were assigned randomly by the instructor, and in the second class, the job fair approach was used. These teams were used for two major projects that took place over the semester, one involving the use of the CAPSIM simulation and the other was a business study requiring research, an interview and a class presentation of their findings. Twenty percent of their course grade was earned on each project. Assessment of the effectiveness of the faux hiring team assignment was assessed in two ways. First, the total team points earned from the simulation and the business projects were compared using a t-test of mean group differences. Results were significant with the faux hiring teams scoring higher than the randomly assigned teams (Table 1). Second, at the end of the semester, students were asked to fill out an anonymous online survey about their team experience. There were 43 respondents to this survey; 19 were from the instructor assigned teams and 24 were from the faux hiring teams. The results show that the faux hiring teams had stronger scores on shared goals, had fewer conflicts, and greater cohesiveness (Table 2).

Table 1: Results for Actual Grades

\begin{tabular}{|c|c|c|c|}
\hline Measurement & t score & Significance & \multicolumn{1}{c|}{ Conclusion } \\
\hline Differences in Total Grade & 2.13 & .017 & $\begin{array}{l}\text { Faux hiring team did much better on final report and } \\
\text { also had significantly lower variance between teams in } \\
\text { the faux hiring class. (n=6 for each class) }\end{array}$ \\
\hline
\end{tabular}


Table 2: Self-report Reflections for In Class Experimentation with Faux Hiring

\begin{tabular}{|l|c|c|l|}
\hline \multicolumn{1}{|c|}{ Item } & $\mathbf{t}$-value & Significance & \multicolumn{1}{c|}{ Conclusion } \\
\hline $\begin{array}{l}\text { I feel that my team was a group that had the same } \\
\text { goals as far as performance. }\end{array}$ & 2.23 & .013 & $\begin{array}{l}\text { The faux hiring team had more goal } \\
\text { agreement. }\end{array}$ \\
\hline My team worked well together overall. & .74 & .33 & No difference in teams \\
\hline $\begin{array}{l}\text { There were conflicts with my team in terms of } \\
\text { decisions. }\end{array}$ & 1.22 & .14 & No significant differences \\
\hline $\begin{array}{l}\text { There was an equivalent amount of time dedicated } \\
\text { by my team members. }\end{array}$ & .77 & .22 & No significant differences \\
\hline My team had a lot of conflicts. & -1.7 & .047 & The faux hiring team had less conflicts. \\
\hline I feel we had a good mix of talents and abilities. & 1.04 & .15 & No significant differences \\
\hline The team was generally cohesive. & 1.94 & .03 & More team cohesion in faux hiring teams \\
\hline The team had serious commitment issues. & -.30 & .33 & No significant differences \\
\hline
\end{tabular}

$\mathrm{n}=43$

Scale: $1=$ strongly disagree, $2=$ disagree, $3=$ =neither agree not disagree, $4=$ agree, $5=$ strongly agree

\section{DISCUSSION}

This methodology has presented a team selection method that combines student input with instructor preferences and results in teams that work together better than if they are randomly formed. This selection method yields improved team satisfaction, cohesiveness and performance.

It is, in essence, informed team self-selection. This method has enhanced team goal achievement, reduced conflicts and improved cohesiveness. It also provides a simulated 'hiring' process that benefits students by providing practice interviewing and presenting themselves in a positive light. This faux hiring process simulates the real world difficulties in finding the best members for the team. The use of a team contract also helps in the formation process by increasing communication on goals and objectives, thus creating better 'buy-in' by the team members. The peer evaluation process also provides a measure of accountability for each member. The measures of success for this study were promising. Of course, any class has its own 'personality' and these results are too small in scope for generalizations. While the class age, gender mix, ethnicities, GPA's and the mix of majors were similar, there are, of course, class culture and personality differences that could play into the findings. However, it is an effective means of team assignment and evaluation that has been used over the past several years with noticeable improvement in team cohesion. There have been fewer instances of emergency team meetings where the students are in serious conflict. It is applicable to any team situation where significant team projects justify the one and a half class periods that need to be dedicated to the team assignment and forming process. This methodology not only indicates that it may enhance team cohesiveness and performance; it also provides valuable experience for the students in the interviewing and self-promotion process they will encounter in their job searches. It would also be appropriate to apply this technique in courses where there would be a focus on interviewing and job placement. The value of the faux hiring process, as a contributor to students' experiences, is an important benefit that justifies the time dedicated to the activity. Further testing of this methodology over a larger sample size and over multiple semesters is the next step in research to validate the successfulness of the method. Greater generalizeability should also be examined using different courses with a variety of grade proportion allocations. The result of successful experiences in student teams is an important precursor to their teamwork when they join the workforce. This process increases their likelihood of a positive attitude toward teamwork in the future.

\section{AUTHOR INFORMATION}

Michelle Lane is an Assistant Professor of management at Western Kentucky University. She has her PhD. in Operations management from the University of South Carolina. Research interests and publications are in the areas of entrepreneurship, social entrepreneurship and supply chain management. She also has publications in the examination of institutional strategy in emerging markets. Her supply chain research examines the use of social media in supply chain integration. Her social entrepreneurship work is concentrated on the measurement of performance and the examination of animal welfare entrepreneurs. E-mail: Michelle.Lane@wku.edu 


\section{REFERENCES}

1. Arien, N.J. (20010. Working Together. San Francisco: Bernett-Koehler.

2. Ashraf, M. (2004). A critical look at the use of group projects as a pedagogical tool. Journal of Education for Business, 79(4), 213-216.

3. Bacon, D. R., Stewart, K.A. and Silver, W.S. (1999). Lessons from the best and worst student team experiences: How a teacher can make the difference. Journal of Management Education, 23(5), 467-488.

4. Brandyberry, A.A. and Bakke, S.A. (2006). Mitigating negative behaviors in student project teams: An information technology solution. Journal of Information Systems Education 17(2), 195-209.

5. Chen, G., Donahue, L. and Klimoski, R. (2004). Training undergraduates to work in organizational teams. Academy of Management Learning and Education 3(1), 27-40.

6. Connerley, M.L. and Mael, F.A. (2001). The importance and invasiveness of student team selection criteria. Journal of Management Education, 25(5), 471

7. Fish, L. (2007). Graduate student project: Operations management product plan. Journal of Education for Business, 83(2), 59-63, 65-71.

8. Hansen, R.S. (2006). Benefits and problems with student teams: Suggestions for improving student team projects. Journal of Education for Business, 82(1), 11-19.

9. Koppenhaver, G.D. and Shrader, C.B. (2003). Structuring the classroom for performance: Cooperative learning with instructor assigned teams. Decision Sciences Journal of Innovative Education 1(1), 1-21.

10. McCloskey, D. (2004). Adding realism to the formation, management and evaluation of project teams. Journal of Information Systems Education, 15(1), 9-11.

11. O'Connor, D. and Yballe, L. (2007). Team leadership: critical steps to great projects. Journal of Management Education, 31(2), 292-312.

12. Stevens, M.A. and Campion, M.J. (1994). Staffing work teams: Development and validation of a selection test for teamwork settings. Journal of Management, 25, 207-228.

13. Wilcoxson, L.E. (2006). "Its not fair!": Assessing the dynamics and resourcing of teamwork. Journal of Management Education, 30(6), 798-808.

14. Williams, S.K. and Anderson, J.S. (2008). Teams Lab: Promoting effective teamwork in operations management classes. Decision Sciences Journal of Innovative Education 6(1), 159-166.

15. Winter, J.K., Waner, K.K. and Neal-Mansfield, J.C. (2008). Team climate and productivity for similar majors versus mixed majors. Journal of Education for Business 84(3), 265-269.

16. Young, C.B. and Henquinet, J. A. (2000). A conceptual framework for designing group projects. Journal of Education for Business, 76(1), 56-60. 